\title{
Preliminary Results of Kansas State University Dense Plasma Focus
}

\author{
A. E. Abdou, Mohamed I. Ismail, Amgad E. Mohamed, S. Lee, S. H. Saw, and R. Verma
}

\begin{abstract}
Kansas State University (KSU) dense plasma focus (DPF) is a $2.5-\mathrm{kJ}$ DPF machine newly commissioned at the Plasma Radiation Physics Laboratory at KSU. The machine was designed to be used as a multiradiation source for applications in nuclear science and engineering. Neutrons are emitted from deuterium-deuterium (D-D) fusion reactions during high-power electric discharges at $17 \mathrm{kV}, 140 \mathrm{kA}$, and 5 mbar. The machine circuit parameters were calculated using the short-circuit test. The emitted neutrons were measured using several radiation detection techniques. The 2.45-MeV characteristic D-D neutron energy was confirmed using the time-of-flight technique using a BC-418 plastic scintillator. The maximum neutron yield was roughly measured to be $2.8 \times 10^{8}$ neutrons per shot using a set of BTI BD-PND bubble detectors. Moreover, the neutron yield variation with pressure was measured and compared with the computed neutron yield using Lee model. Finally, the measured current showed good agreement with Lee six-phase model.
\end{abstract}

Index Terms-Dense plasma focus (DPF), Lee model, multiradiation source, neutron yield.

\section{INTRODUCTION}

A DENSE PLASMA FOCUS (DPF) is a pulsed-power device capable of producing short-lived (tens of nanoseconds), hot $\left(T_{i} \sim\right.$ in kiloelectronvolts $)$, and dense $\left(>10^{19} \mathrm{~cm}^{-3}\right)$ plasma. The machine was independently discovered in the early 60s by Mather in the USA and Filippov in the former Soviet Union [1]. The main difference between Mather and Filippov machine types is the inner electrode aspect ratio (the aspect ratio is defined as the diameter $d$ to the axial length $z$ ). The DPF can be utilized as a multiradiation source capable of simultaneously producing different types of radiation such as fusion neutrons $(\sim 2.45 \mathrm{MeV}$ from deuterium-deuterium (D-D) or $14.1 \mathrm{MeV}$ from deuterium-tritium (D-T) fusion reactions), hard X-rays

Manuscript received October 18, 2011; revised May 30, 2012; accepted July 5, 2012. Date of publication August 23, 2012; date of current version October 5, 2012. This work supported by the Department of Mechanical and Nuclear Engineering, Kansas State University.

A. E. Abdou, M. I. Ismail, and A. E. Mohamed are with the Department of Mechanical and Nuclear Engineering, Kansas State University, Manhattan, KS 66506 USA (e-mail: aeabdou@ksu.edu; mismail1@ksu.edu; engaesm@ksu.edu).

S. Lee and S. H. Saw are with the INTI International University, 71800 Nilai, Malaysia, and also with the Institute for Plasma Focus Studies, Melbourne, Vic. 3148, Australia (e-mail: leesing@optusnet.com.au; sorheoh. saw@newinti.edu).

R. Verma is with the Pulsed Power Group, Institute for Plasma Research, Gandhinagar 382 428, India (e-mail: rishiv9@gmail.com).

Color versions of one or more of the figures in this paper are available online at http://ieeexplore.iee.org.

Digital Object Identifier 10.1109/TPS.2012.2209682

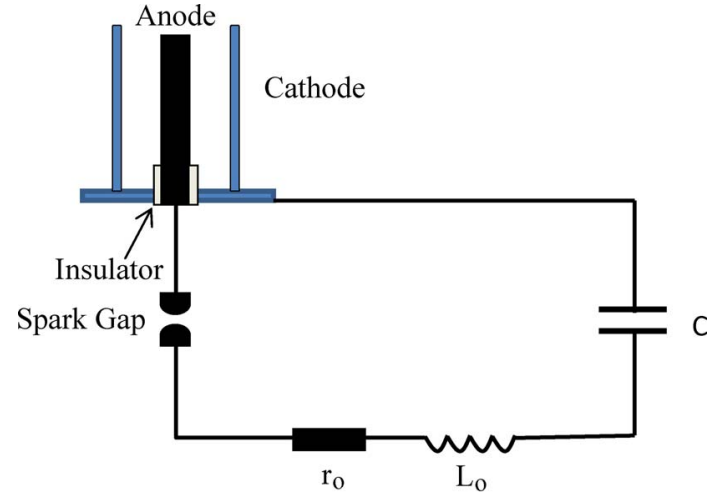

Fig. 1. Schematic for the DPF circuit.

( $\sim$ hundreds of kiloelectronvolts generated as a result of upstream electron beam hitting the bottom of the anode hole), ion and electron beams ( $\sim$ in megaelectronvolts, downstream ion beam and upstream electron beam accelerated as a result of the developed electric field at the end of the compression phase), and finally, electromagnetic radiation ( $\sim$ in gigahertz). The KSU-DPF experimental and computational (using Lee model [2], [3]) results will be discussed.

\section{APPARATUS}

The KSU-DPF has a Mather's type geometry. The machine uses an Aerovox capacitor $\left(C=12.5 \mu \mathrm{F}, L=40 \mathrm{nH}, V_{\max }=\right.$ $20 \mathrm{kV}$, and $I_{\max }=200 \mathrm{kA}$ ). A $35-\mathrm{kV} 8-\mathrm{kJ} / \mathrm{s}$ General Atomics power supply is used as a charger. The capacitor is connected to the electrodes through a TDI1-200 kA/25 kV, 10-ns-jitter, thyratron switch. The central anode is a hollow cylinder with $117-\mathrm{mm}$ height from the cathode base and 7.5-mm radius. Various central electrodes made from copper, brass and stainless steel with straight and tapered geometries were manufactured and will be used to characterize the radiation output of the machine. The central electrode is surrounded by a squirrel-cage cathode consisting of six equally spaced brass bars with $25-\mathrm{mm}$ radius and $120-\mathrm{mm}$ height. At the base, the anode is electrically insulated using a Pyrex glass tube with 68-mm height and 1.6- $\mathrm{mm}$ thickness; the active Pyrex height is $15 \mathrm{~mm}$. A schematic of the experiment is shown in Fig. 1. Several diagnostics were used to measure the machine electrical parameters (i.e., Rogowski coil and high-voltage (HV) probe) and radiation output (plastic scintillators, LiI scintillator, ${ }^{3} \mathrm{He}$ detector with Bonner spheres, and bubble detectors were used for neutron measurements; plastic scintillators and BPX photodiodes were used for X-ray measurements). 


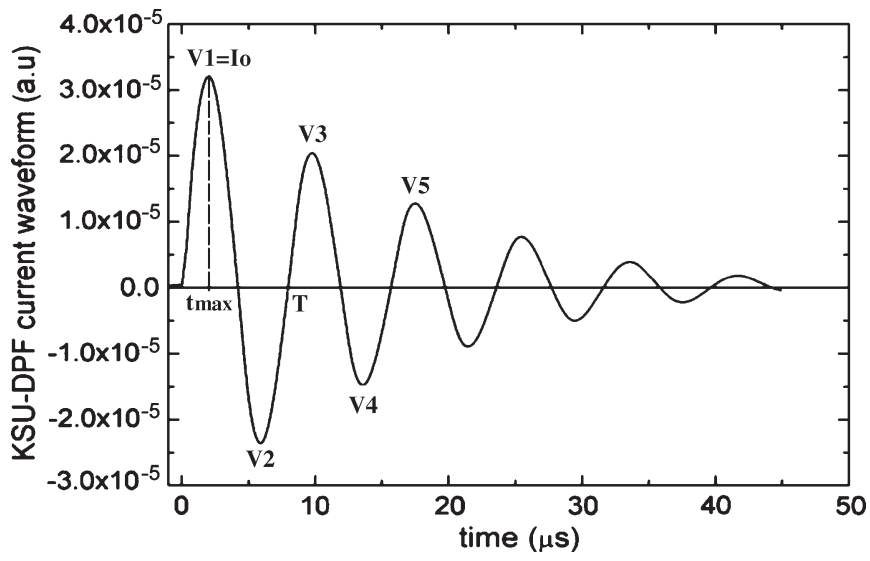

Fig. 2. Measured waveform for the short-circuit test at $17 \mathrm{kV}$.

\section{OpERATION AND DETERMinAtion OF STATIC PARAMETERS}

The short-circuit test is performed to accurately measure the machine static parameters, i.e., $L_{o}$ (circuit inductance in nanohenries), $r_{o}$ (circuit resistance in milliohms), and $C_{o}$ (circuit capacitance taken as $12.5 \mu \mathrm{F}$ ). During the test, the anode was connected directly to the cathode (short circuited using an aluminum circular disk in place of the central electrode connecting the cathode base). The circuit can be electrically represented as a series $R L C$ circuit. Usually, the DPF equivalent circuit in this case is considered to be underdamped [4], i.e., $r_{0} \ll 2 \sqrt{\left(L_{0} / C_{0}\right)}$.

The short-circuit test was done at $17 \mathrm{kV}$ producing the damped sinusoidal waveform shown in Fig. 2. With some approximations, the static parameters (i.e., $L_{o}$ and $r_{o}$ ) and peak current $I_{o}$ are calculated from the following equations [5]:

$$
\begin{aligned}
L_{0} & =\frac{T^{2}}{4 \pi^{2} C_{0}} \\
r_{0} & =-\left(\frac{2}{\pi}\right)(\operatorname{lnf})\left(\frac{L_{0}}{C_{0}}\right)^{0.5} \\
I_{0} & =\frac{\pi C_{0} V_{0}(1+f)}{T}
\end{aligned}
$$

where $f$ is the average value of the reversal ratio obtained from the peaks in Fig. 2.

Hence

$$
\begin{aligned}
f_{1} & =\frac{V_{2}}{V_{1}} \quad f_{2}=\frac{V_{3}}{V_{2}} \quad f_{3}=\frac{V_{4}}{V_{3}} \quad f_{4}=\frac{V_{5}}{V_{4}} \\
f & =\frac{\left(f_{1}+f_{2}+f_{3}+f_{4}\right)}{4} .
\end{aligned}
$$

The KSU-DPF average reverse ratio was calculated to be $f=$ 0.7974 . The discharge period $T=7.85 \mu$ s was calculated by averaging over the first three periods in Fig. 2.

The machine static and discharge parameters are estimated to be $L_{o}=124.94 \mathrm{nH}, \quad r_{o}=14.4 \mathrm{~m} \Omega$, and $I_{\text {peak }}($ discharge peak current $)=152.8 \mathrm{kA}$ operating at $17 \mathrm{kV}$.

During the discharge, the time derivative of the discharge current is measured using a calibrated Rogowski coil $(4.78 \mathrm{E}+6 \mathrm{kA} / \mathrm{V})$ and the discharge voltage is measured using a
NorthStar HV probe (100 kVdc and $80 \mathrm{MHz}$ ) The output of the Rogowski coil and the HV probe are connected using a triaxial cable to a 7000 series Tektronix DPO oscilloscope inside a Faraday cage. The time derivative of the current is numerically integrated using the DPO oscilloscope to obtain the current.

Typical signals for current derivative, voltage, and the numerically integrated current are shown in Fig. 3(a)-(c). An increase in focusing time is noticed with the increase in gas pressure, which is consistent with previous literature work [6], [7]. The pressure dependence of pinch time [see Fig. 3(d)] agrees with the scaling consideration based on the speed factor [8].

\section{TOF Neutron MEAsurements}

In DPF, the time-of-flight (TOF) technique is used to give information about the time-resolved neutron energy spectra [9]. This technique is always performed using a scintillation detector-photomultiplier (PMT) system to register the timeresolved hard X-ray and neutron pulses.

The KSU-DPF TOF detector is a BC-418 plastic scintillator optically connected to HAMAMATSU H7195 PMT. The electrical output is connected to the DPO oscilloscope inside a Faraday cage using a triaxial cable to reduce the signal noise. The scintillator-PMT system was placed at a distance of $3 \mathrm{~m}$ away from the DPF device at $90^{\circ}$ from the machine vertical axis. Measuring the TOF was done using the time difference between the X-ray and neutron pulses shown in Fig. 4 [10].

The two spikes shown in Fig. 4 represent the hard X-ray signal, which reached the scintillator first followed by the neutron signal 138.74 ns later. The estimated neutron velocity based on the measured TOF is $21.62 \mathrm{E}+6 \mathrm{~m} / \mathrm{s}$, and the corresponding neutron energy is $2.447 \mathrm{MeV}$.

\section{Neutron Yield Measurements:}

The neutron generation mechanism is still debated in the pinched plasma discharge (Z-pinch, X-pinch, and plasma focus) communities. Thermonuclear reaction, beam target, and gyrating particle models were all proposed as possible neutron generation mechanisms, but the most accepted mechanisms are the thermonuclear reactions and ion beam-target fusion where total neutron yield $Y=Y_{\mathrm{th}}+Y_{b-t}[1]$.

The neutron yield was estimated using a BTI BD-PND bubble detector located $17.5 \mathrm{~cm}$ away from the center electrode at an axial direction. The dose equivalent of the BD is $6.7 \mathrm{~b} / \mathrm{mrem}$, given by the manufacturer, whereas its conversion factor is $3.48 \mathrm{E}-05\left(\mathrm{mrem} / \mathrm{ncm}^{-2}\right)$. Using this conversion factor, the maximum neutron yield obtained at a single shot was $2.8 \mathrm{E} 8$ on axis; 17 bubbles were obtained at that shot. A series of shots were taken at different deuterium pressures ranging from 1 to 9 mbar, and the neutron yield was measured using a ${ }^{3} \mathrm{He}$ detector located $60 \mathrm{~cm}$ away from the plasma focus at a radial direction; the ${ }^{3} \mathrm{He}$ detector was calibrated by an americium/beryllium (Am/Be) neutron source. Fig. 5 shows the dependence of the neutron yield on the deuterium pressure compared with the theoretical neutron yield, as calculated using Lee model [11]. 

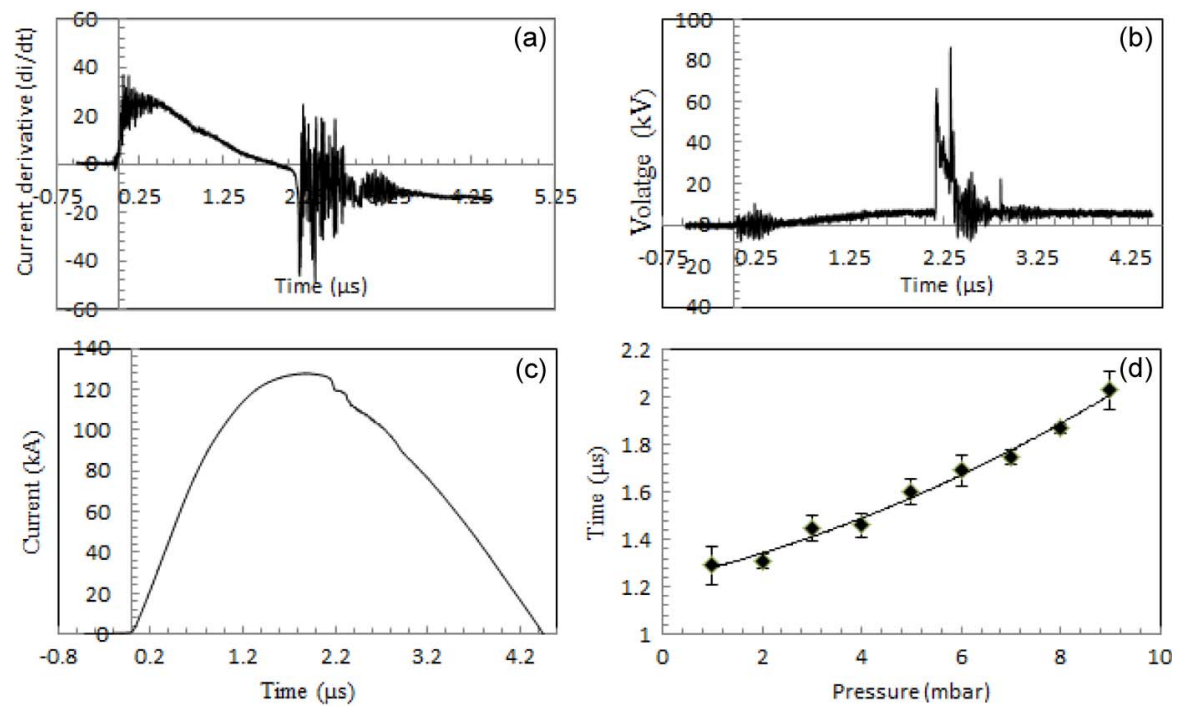

Fig. 3. Typical signals for (a) current derivative, (b) voltage across the electrodes, (c) current integrated by the oscilloscope, and (d) dependence of focusing time on gas pressure.

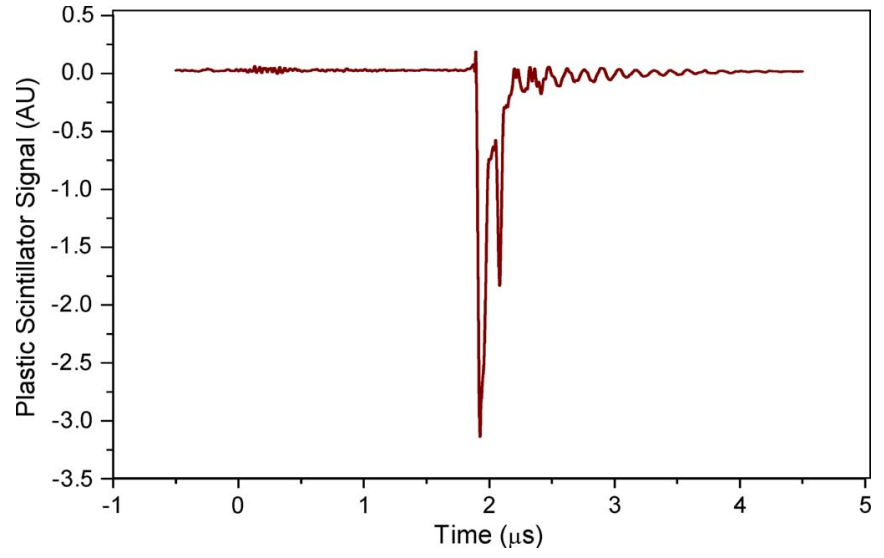

Fig. 4. Output signal of the PMT placed $90^{\circ}$ and $3 \mathrm{~m}$ away.

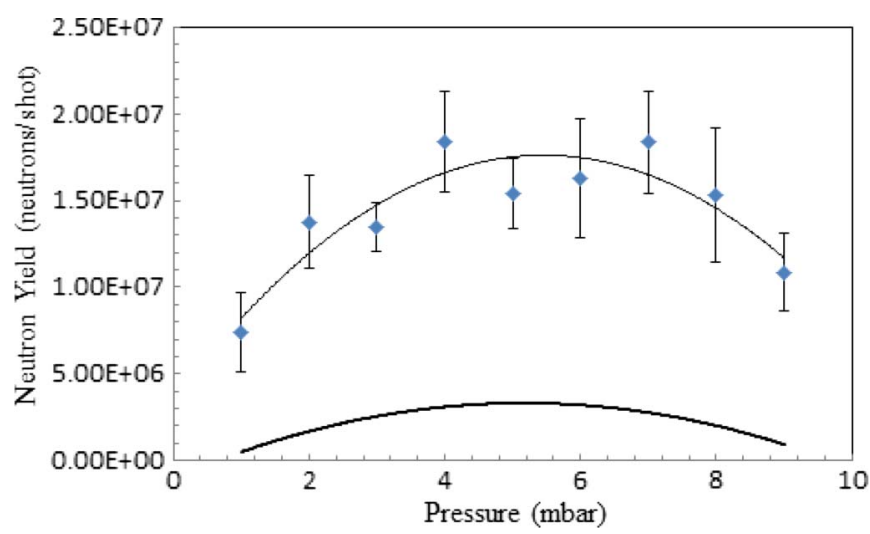

Fig. 5. Neutron yield dependence on pressure.

A recent detailed comparison has shown good agreement of the measured neutron yield with that computed from the Lee model code [11] in the case of the Chilean PF-400J and the Mexican FN-II. These two machines have been identified as T1 low-inductance machines [12]. These results and those of other typical T1 machines, including NX2 and PF1000, show that the measured neutron yield [3] of these T1 machines agrees with the yield computed from the Lee model code to within a factor of 2. Reviewing these results in one glaring example in which the measured yield [13] greatly exceeds the yield computed from the Lee model code. Reference [3, Table I] is that of the UNU ICTP PFF. For this machine, the measured yield is $1 \mathrm{E}+8$ neutrons per shot [13], whereas that computed from the Lee model code (see [3, Table I]) is $2 \mathrm{E}+7$ neutrons per shot.

The measured yield is greater than the computed yield by a factor of 5 . The UNU ICTP PFF is a high-inductance $\left(L_{0}=114 \mathrm{nH}\right) \mathrm{T} 2$ machine. We conclude from this review of measured and computed data that, while T1 (low-inductance) machines show good agreement (within a factor of 2) between the measured (optimized) and computed (from the Lee model code) neutron yields, the measured and computed neutron yields of T2 (high-inductance) machines as represented by the UNU ICTP PFF and the KSU-DPF have measured yields that exceed the computed yield by a typical value of 5 . This conclusion may be significant in further elucidating the neutron production mechanism pertaining to beam target and to further processes that may involve the instabilities of the pinch processes.

\section{Fitting KSU-DPF With LeE Model}

The KSU-DPF is characterized by its high inductance and has been classified as T2 type. It is noticed that the currentwaveform signals have an extended dip beyond the regular dip. Such signals are fitted with the six-phase Lee model [12], an extension to the five-phase model by adding anomalous resistance terms to represent the developed instabilities during the pinch phase. Fig. 6 shows the fitted KSU-DPF current signal to the six-phase Lee model.

\section{CONCLUSION}

The KSU-DPF short-circuit test has shown that it is a highinductance machine, i.e., $L_{o} \approx 125 \mathrm{nH}$. The current signals, obtained using a Rogowski coil, have an extended dip beyond 


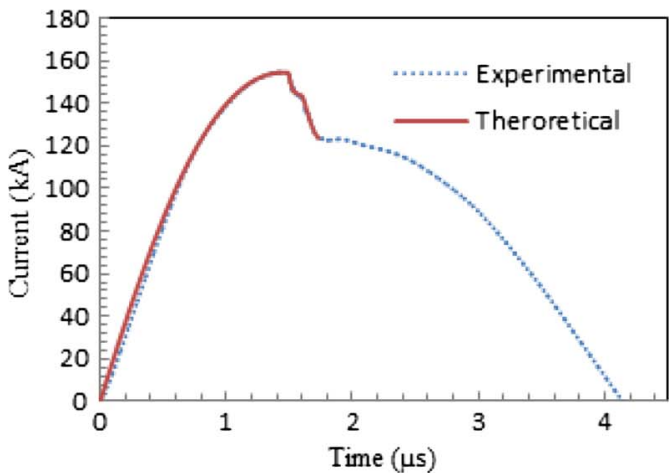

(a)

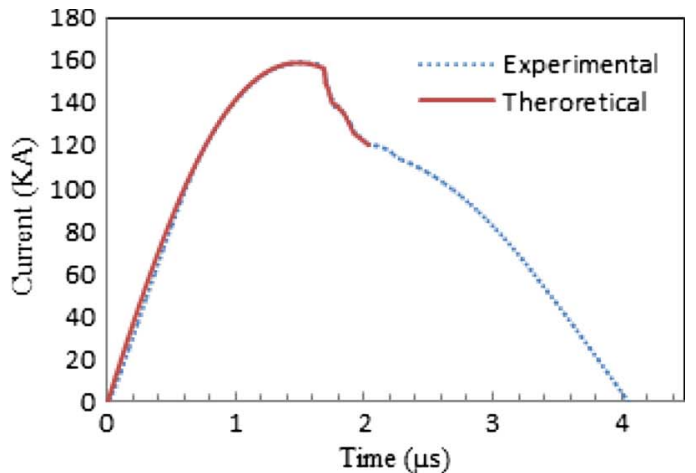

(b)

Fig. 6. Fitting of (a) 3-mbar deuterium. (b) Five-mbar deuterium current signal to the six-phase Lee model. (Fitting stopped at 1.71 and 1.93 , respectively.)

the regular one, and they were fitted using the six-phase Lee model. Using a scintillator-PMT system placed $3 \mathrm{~m}$ away from the focus at $90^{\circ}$, the neutron energy was confirmed verified to be the characteristic 2.45-MeV D-D fusion energy. Analyzing many shots at different deuterium pressures showed that the pinch occurred later on time as the pressure increases. Whereas, for the pressure range from 1- to 9-mbar deuterium, the pinch time varied from 1.29 to $2.03 \mu \mathrm{s}$.

The maximum neutron yield measured in one shot, as estimated by the bubble detector at an axial direction, was $2.8 \mathrm{E} 8$. The maximum neutron yield was obtained at the optimum pressure of 5 mbar. The comparison between the experimental and computed neutron yields indicated that the optimum pressure has reasonable agreement between the computed and measured ones, the falloff on both sides also has reasonable agreement, and finally, the measured median values have around 5 times higher neutron yield than the computed in the region near the optimum. This 5 times excess discrepancy in the measured neutron yield for the KSU-DPF (a high-inductance T2 PF) is also found for the other published T2 machine, i.e., the UNU ICTP PFF, whereas other machines, all low-inductance $\mathrm{T} 1$, have closer agreement (within a factor of 2) between the measured and computed neutron yields.

\section{REFERENCES}

[1] A. Bernard, H. Bruzzone, P. Choi, H. Chuaqui, V. Gribkov, J. Herrera, K. Hirano, A. Krejci, S. Lee, C. Luo, F. Mezzetti, M. Sadowski, H. Schmidt, K. Ware, C. S. Wong, and V. Zoita, "Scientific status of plasma focus research," J. Moscow Phys. Soc., vol. 8, pp. 93-170, 1998.

[2] S. Lee, Radiative Dense Plasma Focus Computation Package: RADPF2011. [Online]. Available: www.intimal.edu.my/school/fas/ UFLF
[3] S. Lee and S. H. Saw, "Neutron scaling laws from numerical experiments," J. Fusion Energy, vol. 27, no. 4, pp. 292-295, 2008.

[4] M. Frignani, "Simulation of gas breakdown and plasma dynamics in plasma focus devices," Ph.D. dissertation, Alma Mater Studiorum Università degli Studi di Bologna, Rimini, Italy, 2007.

[5] S. H. Saw, S. Lee, F. Roy, P. L. Chong, V. Vengadeswaran, A. S. M. Sidik, Y. W. Leong, and A. Singh, "In situ determination of the static inductance and resistance of a plasma focus capacitor bank," Rev. Sci. Instrum., vol. 81, no. 5, p. 053 505, May 2010.

[6] C. Moreno, A. Clausse, H. Bruzzone, J. Martínez, R. Llovera, and A. Tartaglione, "Small-chamber $4.7 \mathrm{~kJ}$ plasma focus for applications," in Proc. Plasma Phys._IX Latin Amer. Workshop, 2001, vol. 563, pp. 276-281.

[7] C. Moreno, H. Bruzzone, J. Martínez, and A. Clausse, "Conceptual engineering of plasma focus thermonuclear pulsors," IEEE Trans. Plasma Sci., vol. 28, no. 5, pp. 1735-1741, Oct. 2000.

[8] S. Lee and A. Serban, "Dimensions and lifetime of plasma focus pinch," IEEE Trans. Plasma Sci., vol. 24, no. 3, pp. 1101-1105, Jun. 1996.

[9] K. J. Tomaszewski, "Diagnostic system for time-of-flight neutron measurements," in Proc. 16th IAEA Tech. Meeting Res. Using Small Fusion Devices; XI Latin Amer. Workshop Plasma Phys. AIP, 2006, vol. 875, pp. 41-44.

[10] V. Siahpoush and M. A. Mohammdi, "Preliminary results of neutron production in Sahand plasma focus device," in Proc. 2nd Int. Conf. Nucl. Sci. Technol. Iran, Shiraz Univ., Apr. 27-30, 2004, pp. 27-30.

[11] S. Lee, S. H. Saw, L. Soto, S. P. Moo, and S. V. Springham, "Numerical experiments on plasma focus neutron yield versus pressure compared with laboratory experiments," Plasma Phys. Control. Fusion, vol. 51, no. 7, p. 075006, Jul. 2009.

[12] S. Lee, S. H. Saw, A. E. Abdou, and H. Torreblanca, "Characterizing plasma focus devices-Role of the static inductance-instability phase fitted by anomalous resistances," J. Fusion Energy, vol. 30, no. 4, pp. $277-$ 282, Aug. 2011.

[13] S. Lee, T. Y. Tou, S. P. Moo, M. A. Eissa, A. V. Gholap, K. H. Kwek, S. Mulyodrono, A. J. Smith, Suryadi, W. Usada, and M. Zakaullah, "A simple facility for the teaching of plasma dynamics and plasma nuclear fusion," Amer. J. Phys., vol. 56, no. 1, pp. 62-68, Jan. 1988.

Authors' photographs and biographies not available at the time of publication. 